\title{
Biased mu-opioid receptor agonists confer analgesia with reduced side effects
}

\author{
Chloe Gui, Sean Wong
}

\section{ABSTRACT}

Opioids are considered mainstay treatments for acute and terminal pain. In recent decades, however, overprescription and the increasing prevalence of illicit opioids has propelled North America into a state of "opioid crisis." Along with the analgesic benefits, opioid use also commonly induces a number of side effects. Respiratory depression is an especially dangerous and potentially lethal example. The development of painkillers with improved safety profiles is thus a priority. Downstream to the mu-opioid receptor, which is responsible for the analgesic effects of opioids, $\beta$-arrestin-2 signaling has been suggested to be important for the manifestation of side effects, including respiratory depression. Two novel muopioid receptor agonists, TRV130 and PMZ21, have recently been reported to preferentially promote $\mathrm{G}$ protein-coupling over $\beta$-arrestin-2 signaling, thereby promoting analgesia with reduced side effects. TRV130 has been found in clinical trials to be more potent than morphine but safer in the setting of acute moderate-to-severe pain and is currently under New Drug Application review in the U.S. PMZ21 has shown promising and unique pain-relieving effects in mouse models, but further investigation is warranted to examine whether its therapeutic effects and safety profile are translatable to humans.

\section{INTRODUCTION}

Opioids are compounds that bind opioid receptors and include: morphine and codeine, which are derived from poppy seeds (Papaver somniferum); semi-synthetic compounds such as heroin and oxycodone; and synthetic compounds including methadone, fentanyl, and propoxyphene. ${ }^{1}$ Opioid receptors are transmembrane G protein-coupled receptors (GPCRs) that are classified into mu, delta, kappa, and nociception subtypes. ${ }^{1}$ The analgesic and reinforcing effects of opioids are primarily mediated by mu-opioid receptors (MORs), which are widely-expressed in the central and peripheral nervous systems and in the gastrointestinal tract., ${ }^{2,3}$ Medically, opioids are used for pain relief, but chronic use and abuse can lead to physical dependence. One of the biggest challenges in treating chronic pain with opioids is providing sufficient symptom relief while minimizing the risk of dependency and other side effects. Here, we examine promising novel opioids that are simultaneously biased towards analgesic signaling pathways and against pathways conferring side effects, thereby providing pain relief with fewer adverse events (AEs). We will also briefly discuss a computational structure-based approach for novel opioid discovery.

\section{CURRENT LIMITATIONS OF OPIOIDS}

Though opioid addiction has been described as early as the sixteenth century in Europe, in recent years, the prevalence of opioid drug abuse in both Canada and the United States has increased, leading to an increase in opioid-related hospitalizations and deaths.-6 This epidemic has been attributed to multiple factors including over-prescription, the rise in illicit heroin and fentanyl, and aggressive marketing by pharmaceutical companies. ${ }^{6,7}$ Chronic use of opioids can lead to physical dependence, and abrupt abstinence can trigger withdrawal symptoms that include drug cravings, anxiety, diaphoresis, tachycardia, and gastrointestinal distress. ${ }^{8}$ Side effects of these medications also include sedation, dizziness, gastrointestinal distress, tolerance and respiratory depression. ${ }^{8}$ The latter is particularly dangerous and is the primary cause of opioid-related overdose and death. ${ }^{8,9}$ Furthermore, patients on opioids must be tapered off slowly to avoid withdrawal..$^{10}$ MOR signaling is responsible for both the analgesic and side effects of opioids. Hence, side effects of MOR agonists are considered to be "on target" effects and have traditionally been regarded as inevitable disadvantages of MOR-targeting opioids. These AEs also limit the maximum dosage of morphine and other opioids. ${ }^{2}$

\section{G PROTEIN-BASED OPIOIDS AND TRV-130}

In recent years, it has been demonstrated that different intracellular pathways can be induced by activation of a single GPCR receptor. Different agonists may demonstrate activation bias towards one particular downstream pathway over another. ${ }^{11,12}$ In the case of MORs, studies have shown that some agonists exhibit G protein-coupling bias while others exhibit bias towards alternative pathways such as $\beta$-arrestin signaling. ${ }^{13,14}$ This signaling preference results in distinctive functional selectivity despite having a common receptor. ${ }^{13,14}$ Studies examining MOR signaling have identified $\beta$-arrestins to be key modulators of opioid effects. For instance, $\beta$-arrestin-2 contributes to opioid tolerance by binding to and desensitizing MORs to ligands and $\beta$-arrestin- 1 has been shown to promote ubiquitin-proteasomal degradation of the receptor. ${ }^{13}$ In mice lacking $\beta$-arrestin-2, chronic morphine treatment did not induce tolerance, suggesting that $\beta$-arrestin- 2 is also necessary for opioid tolerance development..$^{15}$ Furthermore, morphine-treated $\beta$-arrestin-2 knockout mice also exhibited less physical dependence, constipation, and respiratory depression than their wild-type littermates, although this effect disappeared at higher doses of morphine. ${ }^{13}$ The analgesic effects of morphine were present and, in fact, enhanced in the $\beta$-arrestin-2 knockout mice. Thus, the $\beta$-arrestin-2 pathway is implicated in major doselimiting AEs but not in analgesia. Respiratory depression associated 
with morphine use persists even after the analgesic response ends, further suggesting that unique signaling pathways are responsible for the therapeutic effects and side effects of opioids. ${ }^{14,16}$ In line with this conclusion, $\beta$-arrestin recruitment is associated with a narrower therapeutic window due to increased risk of AEs. For example, fentanyl exhibits preference for $\beta$-arrestin signaling over $\mathrm{G}$ protein signaling and has been shown to cause respiratory depression even at low analgesic doses. ${ }^{17}$ Taken together, an opioid biased towards $\mathrm{G}$ protein signaling and against $\beta$-arrestin-2-recruitment could potentially provide pain relief with lessened risks of dependence and respiratory depression.

In 2013, DeWire et al of Trevana Inc reported the discovery of TRV130, a novel G protein-biased MOR-targeting compound exhibiting minimal $\beta$-arrestin- 2 recruitment. To find this compound, the investigators experimentally screened the internal chemical library at Trevana, seeking a compound with high G protein but low $\beta$-arrestin-2 signaling. TRV130, later named Oliceridine or OLINVO, was found to activate $G$ protein-coupling to a similar extent compared to morphine but was $86 \%$ less efficient in recruiting $\beta$-arrestin-2. TRV130 is also notably more potent than morphine and structurally different from known MOR agonists. ${ }^{18}$ In a recent phase IIb clinical trial, TRV130 was reported to provide effective pain relief for patients who had undergone abdominoplasty. Pain relief achieved was significantly greater than that provided by placebo, and TRV130 was found to be significantly more potent than morphine as well. Patients treated with TRV130 were less likely to experience side effects, including nausea, vomiting, and respiratory dysfunction; in other words, TRV130 has a wider therapeutic window and may be safer than morphine. ${ }^{19}$ A press release dated November 7, 2017 announced that phase III trials of intravenous TRV130 for moderate-to-severe acute pain have shown promising results, and a New Drug Application has been submitted to the U.S. Food and Drug Administration (FDA). ${ }^{20}$

\section{STRUCTURE-BASED DISCOVERY OF BIASED OPIOID AGONIST PZM21}

Alongside to traditional experimental screening, virtual screening has become a successful tool for drug discovery. ${ }^{21-24}$ Virtual screening by molecular docking is a computational method that has been used to study ligand-receptor interaction since its invention in 1980. ${ }^{25}$ In molecular docking, algorithms predict conformations of the ligand-receptor complex and subsequently rank these by a score that represents how well the ligand and receptor bind. Subsequently, the rankings of ligands and their conformations allow researchers to shortlist potential candidates for further investigation. Improvements in computational power, improved screening software, and the increased availability of structural data have allowed researchers to approach drug design from a structural perspective. Molecular docking is therefore a virtual screening technique that is cost-effective and potentially more efficient than experimental screening. ${ }^{26}$ Recently, Manglik et al employed this structure-based method of drug discovery to identify a novel G protein-biased opioid, PZM $21 .^{27}$
In the pursuit of superior MOR ligands, Manglik et al studied the binding properties of 3 million compounds in 1.3 million configurations, each against inactive MOR. 2500 compounds with the best docking profiles were manually inspected for novelty and interaction with key residues, and of these, 23 molecules were selected for further testing. After optimizing compounds for affinity, the most potent compound, PZM21, exhibited strong $\mathrm{G}$ protein-coupled activity with no detectable $\beta$-arrestin-2 recruitment. PZM21 was revealed to be highly specific for MOR, showing no kappa- or nociception-opioid receptor activity and only weak delta-opioid receptor activation. In mouse studies, PZM21 conferred analgesia in a hotplate assay, which assesses higher-level central nervous system and spinal nociceptive circuits, but no pain relief in the tail-flick assay, which assesses spinal reflexes. These experiments suggest that PZM21 selectively blocks the affective component of pain, a distinction novel among opioids. Furthermore, mice treated with PZM21 did not exhibit respiratory depression whereas morphine at equi-analgesic doses suppressed respiration. Mice given PZM21 also did not display a phenotype consistent with reward circuit activation, which is linked to reinforcing behaviours and addiction. On the other hand, mice given morphine demonstrated acute hyperlocomotive responses and preferred to spend time in locations associated with morphine administration, both markers of reward circuit activation. ${ }^{27}$ Overall, PZM21 is a novel biased opioid agonist demonstrating strong $G$ protein signaling preference over $\beta$-arrestin- 2 recruitment, resulting in potent analgesic benefits with reduced AEs.

\section{FUTURE DIRECTIONS AND SUMMARY}

Both TRV130 and PZM21 are promising G protein-biased MOR ligands with fewer side effects compared to other opioids. The mechanisms of these drugs suggest that robust activation of the $G$ protein signaling pathway and avoidance of $\beta$-arrestin- 2 recruitment broadens the therapeutic window and increases the safety of opioid compounds. Interestingly, TRV130 and PZM21 exhibit unique signaling despite sharing a bias for G protein-coupling. Notably, PZM21 induces less respiratory depression than TRV130, which causes significant depression, though to a lesser extent than morphine. PZM21 also has little effect on the reflexive pain circuit and provides primarily afferent pain relief. ${ }^{27}$ Thus, structure-based drug discovery, as demonstrated by the discovery of PZM21, may be useful for identifying novel ligands and elucidating novel pathways. PZM21 remains to be clinically validated as an painkiller, however, and its side effects and risks need to be further investigated at analgesic dose. Currently, TRV130 is awaiting FDA New Drug approval in the U.S. Its wider therapeutic window, lessened AEs, and demonstrated effectiveness in treating moderate-to-severe acute pain making it an attractive alternative to current opioid medications in some clinical settings.

\section{REFERENCES}

1. Rosenblum A, Marsch L, Herman J, et al. Opioids and the treatment of chronic pain: controversies, current status, and future directions. Exp Clin Psychopharmacol. 2008 Oct;16(5):405-16. 
2. Sora I, Takahashi N, Funada M, et al. Opiate receptor knockout mice define $\times$ receptor roles in endogenous nociceptive responses and morphine-induced analgesia. Proc Natl Acad Sci. 1997 Feb 18;94(4):1544-9.

3. Matthes HWD, Maldonado R, Simonin F, et al. Loss of morphineinduced analgesia, reward effect and withdrawal symptoms in mice lacking the $\mu$-opioid-receptor gene. Nature. 1996 Oct; 383(6603):81923.

4. Brownstein MJ. A brief history of opiates, opioid peptides, and opioid receptors. Proc Natl Acad Sci. 1993 Jun;90(12):5391-3.

5. Opioid Overdose Crisis [Internet]. National Institute on Drug Abuse; 2017 Jun [cited 2017 Nov 20]. Available from: https://www.drugabuse. gov/drugs-abuse/opioids/opioid-overdose-crisis.

6. Federal Action on Opioids [Internet]. Government of Canada; 2017 November [cited 2017 November 20]. Available from: https://www. canada.ca/en/health-canada/services/substance-abuse/prescriptiondrug-abuse/opioids/federal-actions.html.

7. Van Zee A. The Promotion and Marketing of OxyContin: Commercial Triumph, Public Health Tragedy. Am J Public Health. 2009 Feb;99(2):221-7.

8. Benyamin R, Trescot A, Datta S, et al. Opioid complications and side effects. Pain Physician. 2008;11:S105-S120.

9. Management of substance abuse: Information sheet on opioid overdose [Internet]. World Health Organization; 2014 Nov [cited 2017 Nov 20]. Available from: http://www.who.int/substance_abuse/ information-sheet/en/.

10. Medically supervised opioid withdrawal during treatment for addiction [Internet]. UpToDate; 2017 Sep 15 [cited 2017 Nov 20]. Available from: http://www.uptodate.com/contents/ medically-supervised-opioid-withdrawal-during-treatment-foraddiction?source $=$ see_link.

11. Rajagopal S, Bassoni DL, Campbell JJ, et al. Biased agonism as a mechanism for differential signaling by chemokine receptors. J Biol Chem. 2013 Oct;288(49):35039-48.

12. Reiner S, Ambrosio M, Hoffmann C, et al. Differential Signaling of the Endogenous Agonists at the $\beta 2$-Adrenergic Receptor. J Biol Chem. 2010 Sep;285(46):36188-98.

13. Raehal KM, Schmid CL, Groer CE, et al. Functional Selectivity at the mu-Opioid Receptor: Implications for Understanding Opioid Analgesia. Pharmacol Rev. 2011 Dec;63(4):1001-19.

14. Raehal KM, Bohn LM. $\beta$-Arrestins: Regulatory Role and Therapeutic Potential in Opioid and Cannabinoid Receptor-Mediated Analgesia. 2014 Mar;219:427-43.

15. Bohn LM, Gainetdinov RR, Lin FT, et al. Mu-opioid receptor desensitization by beta-arrestin-2 determines morphine tolerance but not dependence. Nature. 2000 Dec;408(6813):720-3.

16. Raehal KM, Walker JKL, Bohn LM. Morphine side effects in beta-arrestin-2 knockout mice. J Pharmacol Exp Ther. 2005 Sep;314(3):1195-201.

17. Schmid CL, Kennedy NM, Ross NC, et al. Bias Factor and Therapeutic Window Correlate to Predict Safer Opioid Analgesics. Cell. 2017 Nov;171(5):1165-1175.e13.

18. DeWire SM, Yamashita DS, Rominger DH, et al. A G Protein-Biased Ligand at the $\mu$-Opioid Receptor Is Potently Analgesic with Reduced Gastrointestinal and Respiratory Dysfunction Compared with Morphine. J Pharmacol Exp Ther. 2013 Mar;344(3):708-17.

19. Singla N, Minkowitz HS, Soergel DG, et al. A randomized, Phase IIb study investigating oliceridine (TRV130), a novel $\mu$-receptor G-protein pathway selective ( $\mu$-GPS) modulator, for the management of moderate to severe acute pain following abdominoplasty. J Pain Res. 2017 Oct;10:2413-24.

20. Trevena Reports Third Quarter 2017 Financial Results and Announces New Positive Clinical Trial Data [Internet]. Trevena Inc; 2017 Nov 7 [cited 2017 Nov 20]. Available from: http://www.trevena.com/newsdetails.php?id=186.

21. Grneberg S, Wendt B, Klebe G. Subnanomolar inhibitors from computer screening: A model study using human carbonic anhydrase II. Angew Chemie. 2001 Jan;40(2).

22. Doman TN, McGovern SL, Witherbee BJ, et al. Molecular docking and high-throughput screening for novel inhibitors of protein tyrosine phosphatase-1B. J Med Chem. 2002 May;45(11):2213-21.

23. Schapira M, Raaka BM, Samuels HH, et al. Rational discovery of novel nuclear hormone receptor antagonists. Proc Natl Acad Sci. 2000 Feb;97:1008-13.

24. Boehm HJ, Boehringer M, Bur D, et al. Novel inhibitors of DNA gyrase: 3D structure based biased needle screening, hit validation by biophysical methods, and 3D guided optimization. A promising alternative to random screening. J Med Chem. 2000 Jul;43(14):266474 .

25. Kuntz ID, Blaney JM, Oatley SJ, et al. A Geometric Approach To Macromolecule-Ligand Interactions. J Mol Biol. 1982 Oct;161(2):26988.

26. Meng X-Y, Zhang H-X, Mezei M, et al. Molecular docking: a powerful approach for structure-based drug discovery. Curr Comput Aided Drug Des. 2011 Jun;7(2):146-57.

27. Manglik A, Lin H, Aryal DK, et al. Structure-based discovery of opioid analgesics with reduced side effects. Nature. Nature Publishing Group. 2016 Sep;537(7619):185-90. 